\title{
Research Paper \\ Assessment of Lippia Citriodora Oil Effect on Sleep Disturbances in Ageing: A Double Blind Randomized Controlled Trial
}

\author{
Seyran Mohamadi ${ }^{1} \odot$, Robab Sahaf ${ }^{1} \odot$, Mehrdad Karimi ${ }^{2 *} \odot$, Ahmad Ali Akbari Kamrani ${ }^{1} \odot$, Mohsen Shati ${ }^{1} \odot$, Arash Mira- \\ bzadeh $^{3} \odot$, Gholamreza Ansari ${ }^{1} \odot$
}

1. Department of Aging, Iranian Research Center on Aging, University of Social Welfare and Rehabilitation Sciences, Tehran, Iran.
2. Department of Iranian Medicine, School of Traditional Medicine, Tehran University of Medical Sciences, Tehran, Iran.
3. Department of Psychiatry, Social Determinants of Health Research Center, University of Social Welfare and Rehabilitation Sciences, Tehran, Iran.

\begin{tabular}{|l|l|}
$\begin{array}{c}\text { Use yur devic to scan } \\
\text { and read the article online }\end{array}$ & $\begin{array}{l}\text { Cttation: Mohamadi S, Sahaf R, Karimi M, Akbari Kamrani AA, Shati M, Mirabzadeh A, et al. [Effectiveness of Lemon } \\
\text { Verbena Oil on Sleep Disturbances in Older People: A Double Blind Randomized Controlled Trial (Persian)]. Iranian Journal of } \\
\text { Ageing. 2020; 15(1):54-67. https://doi.org/10.32598/sija.15.1.2234.2 }\end{array}$ \\
doi https://doi.org/10.32598/sija.15.1.2234.2
\end{tabular}

\section{(1) (3)}

Received: 10 June 2018 Accepted: 08 Dec 2019 Available Online: 24 Mar 2020

Key words:

Elderly, Sleep disturbances, Lippia Citriodora oil, Pittsburgh Questionnaire

\section{ABSTRACT}

Objectives The elderly population is increasing globally and as the age increases and the functions of some organs decrease, some problems in elderly age, including sleep problems increase. Sleep disturbances cause neurological, autonomic, and biological changes. Therefore, attention should be paid to the problems that eliciting for the elderly and their families subsequently. The aim of this study was to evaluate the effect of oil on lemon on sleep disorders in the elderly.

Methods \& Materials The study is a stratified design with IRCT2016061528485N1 registration Code and IR.USWR.REC.1395.50 Code of Ethics approved by the Ethics Committee of the University of Social Welfare and Rehabilitation Sciences. A total of 93 subjects ( 45 men and 48 women) were enrolled in the intervention, 22 men in the intervention group, 23 in the control group, 24 in the intervention group and 24 in the control group. Of these, 85 participated in the third stage of the intervention, 43 women (51\%) and 42 men (49\%), 41.17\% in the age group of $74-60$ years and $58.82 \%$ in the age group above 75 years. The Mean \pm SD age of the elderly was $75.88 \pm 9.19$ years, with the lowest age of 61 years and the highest age of 94 years. Outbreaks were hospitalized, departures and deaths. After completing an examination, a score of more than 5 Pittsburgh questionnaires, a visit by a psychiatrist and a self-reported sleep disorder, and considering the medical records, consumables, and entry criteria they were studied according to the randomization tables and divided into intervention and control groups. Validity and reliability of Pittsburgh questionnaire have been studied in various research. The intervention group received oil from the lemon and the inactive oil control group for four weeks, once daily, twice daily in the morning and night, with a drop per nose. No specific side effects were reported. In 3 cases, mild headache was reported, which was associated with a history of sinusitis, and the intervention was either performed or excluded from the tampon.

Results At the beginning of the study, demographic questionnaires, Pittsburgh Sleep Quality Index, and then Pittsburgh Questionnaire 2 and 4 weeks after the beginning of the intervention, were again asked by interview method and the data were analyzed by SPSS V. 23. The tests were one-way variance analysis, follow-up tests, independent samples $T$ and single-sample t-test.

Conclusion Based on the results of the study, the oil to lemon has been shown to improve the sleep disorders of the elderly in the intervention group $(P=0.0003)$ and Effect size $=0.155)$. Sleep disturbances The intervention group improved in the second week and the fourth week after the onset of intervention compared to the control group.

\section{* Corresponding Author: \\ Mehrdad Karimi, PhD.}

Address: Department of Iranian Medicine, School of Traditional Medicine, Tehran University of Medical Sciences, Tehran, Iran.

Tel: +98 (21) 88990837

E-mail: mehrdadkarimi@yahoo.com 


\section{Extended Abstract}

\section{Introduction}

$\mathbf{T}$

he elderly population in the world is increasing on a daily basis. Aging is not a phenomenon that alone causes disease, there is an obvious link between aging and diseases. Aging and sleep-related problems increase mortality from serious illnesses [1].

In the texts of traditional medicine, sleep is defined as follows: Sleep is the return of the instinctual heat to the inward to rest the forces and digest food; in other words, sleep is the cessation of the movement of the soul from the use of the external and natural senses. Sleep has four causes: the material cause of sleep is moderate humidity; the formal cause of sleep is the amount, time, and form of sleep; the actual cause of sleep is the animal soul; and the ultimate cause of sleep is the gathering and return of forces to the inward to seek rest [2]; But modern medicine sources say that sleep and rest is a complex and dynamic physiological state that is necessary for survival and if it is not met, human life is endangered [3] and sleep deprivation causes neurological, autonomic and biological changes [4].

Some elderly may consider sleep-related problems to be natural age-related changes and may not seek appropriate treatment. There are several pharmacological and nonpharmacological treatments for sleep-related problems, including methods such as sleep hygiene, pharmacotherapy, cognitive-behavioral therapies, bright light therapy, complementary and traditional medicine methods (such as hydrotherapy, herbal medicine, acupressure, acupuncture, relaxation, etc.) $[4,10,14-17]$. According to research, sleep problems are solved in $20 \%$ of the elderly with the help of painkillers, anti-anxiety and hypnotic drugs, and for $57 \%$ of the elderly, behavioral therapy methods are used $[3,18]$. When considering medication for a patient with sleep disorders, in addition to the possibility of several diseases, related therapies should also be considered. Some drugs are stimulants and may cause insomnia, including central nervous system stimulants, beta blockers, diuretics, bronchodilators, calcium channel blockers, corticosteroids, decongestants, antidepressants, and thyroid hormones [19].

One of the effects mentioned in the texts that may occur after consuming lippia citriodora (lemon verbena), is sedation and subsequent improvement of sleep disorders. Its properties have been mentioned many times in traditional medicine texts, but in modern medicine, no research has been found that shows the effect of lemon verbena on sleep disorders; So far, no extensive studies have been conducted in the country or abroad on the use of lippia citriodora or its products in the treatment of insomnia, and the studies that have been done -especially at home - have been very brief. Therefore, we decided to investigate the effects of lippia citriodora oil on sleep disorders in the elderly. We hope that the results of this study contain useful information and solutions for the elderly and their caregivers regarding the use of lippia citriodora oil to improve the effects of insomnia.

\section{Methods \& Materials}

The present study was a double-blind randomized controlled clinical trial, which is also called "stratified design". Inclusion criteria were: willingness to participate in the study; age 60 years and older; self-reported sleep disorder at the clinical level and according to the Pittsburgh questionnaire; ability to answer questionnaire items; no history of any allergies to lippia citriodora or any type of verbenaceae; lack of continuous consumption (twice or more per week) of lippia citriodora herbal tea or essence during the past 1 month; no nasal airway obstruction. Exclusion criteria were: inclination to leave the study; depression, anxiety or severe stress during the intervention (for example, due to the death of a loved one); discontinuation or less consumption of lippia citriodora oil by the subject for less than 24 consecutive days before completion of intervention period; showing any allergic reaction to lippia citriodora oil during the research; dependence or addiction to alcohol, drugs and medications; kidney disease; heart failure; respiratory disorders and sleepwake cycle diseases such as respiratory apnea and restless legs syndrome; the use of drugs that affect the sleep-wake cycle (except for previously prescribed drugs).

After evaluating the patients for inclusion criteria, the baseline information including the Pittsburgh Sleep Quality Index and demographic information were asked.

Lippia citriodora oil preparation process: $50 \mathrm{~g}$ of the prepared and approved sample of lippia citriodora is poured into $200 \mathrm{cc}$ of inert oil and then $200 \mathrm{cc}$ of distilled water is added to it and subjected to indirect heat until its water evaporates completely and finally, the plant residue is obtained from the refined oil to the final product of the lippia citriodora oil. The method of using inert oil (placebo) and lippia citriodora oil (intervention) was by dripping a drop of the corresponding oil in the morning and night.

\section{Results}

Descriptive statistics on sleep disorder indices are presented in Table 1. 
Table 1. Descriptive statistics related to sleep disorders index in three stages of sampling

\begin{tabular}{|c|c|c|c|}
\hline Stages & Group & Mean \pm SD & Quantity \\
\hline \multirow{3}{*}{ Sleep disorders before intervention } & Control group & $11.06 \pm 3.44$ & 34 \\
\hline & Intervention group & $11.05 \pm 4.33$ & 40 \\
\hline & Total & $11.05 \pm 3.92$ & 74 \\
\hline \multirow{3}{*}{ Sleep disorders two weeks after the intervention } & Control group & $10.65 \pm 4.62$ & 34 \\
\hline & Intervention group & $8.17 \pm 3.43$ & 40 \\
\hline & Total & $9.31 \pm 4.18$ & 74 \\
\hline \multirow{3}{*}{ Sleep disorders one month after the intervention } & Control group & $12.71 \pm 3.40$ & 34 \\
\hline & Intervention group & $9.20 \pm 4.31$ & 40 \\
\hline & Total & $10.81 \pm 4.27$ & 74 \\
\hline
\end{tabular}

\section{Conclusion}

The aim of this study was to investigate the effect of lippia citriodora oil in comparison with inert oil on sleep disorders in the elderly. At the beginning of the intervention, in a few cases, the problem of headache was mentioned. After examining the problem, it was found that due to the fact that part of the intervention was performed in autumn and winter, and considering that the drops were oily and dripped into the nose, in the elderly with a history of sinusitis these concentrated drops were less absorbed and caused occasional and short-term headaches. Finally, it was decided that either the elderly should be excluded from the study or the oil should be poured on cotton tampons and placed in their noses. In the selection of other subjects, they were asked about their sinusitis or occasional headaches, and if they had a history of these diseases they were excluded from the intervention. The hypothesis was that the mean score of sleep disorders in the elderly in the intervention and control groups before and after the intervention were different. According to the results of the study, the hypothesis was examined and the score of sleep disorders in the elderly in the intervention group decreased compared to the control group. In other words, lippia citriodora oil was effective on sleep disorders in the elderly.

Based on the results of the present study, the use of lippia citriodora oil is recommended as a complementary and uncomplicated treatment for sleep disorders in the elderly. Due to the low price and low complication of herbal treatments and the favorable tendency and mentality of the majority of the elderly to use these treatments, by conducting scientific studies and documenting the desired effects (such as the results of the present dissertation), these treatments can be integrated in conventional medicine and recommended to medical staff and health care system managers.

\section{Ethical Considerations}

\section{Compliance with ethical guidelines}

All ethical principles were considered in this article. The participants were informed about the purpose of the research and its implementation stages; they were also assured about the confidentiality of their information; Moreover, They were allowed to leave the study whenever they wish, and if desired, the results of the research would be available to them.

\section{Funding}

This research did not receive any specific grant from funding agencies in the public, commercial, or not-forprofit sectors.

\section{Authors' contributions}

All authors contributed equally in preparing all parts of the research.

\section{Conflicts of interest}

The authors declared no conflict of interest.

\section{Acknowledgements}

The researchers appreciate the Professors of the Department of Aging of the University of Social Welfare and Rehabilitation Sciences, Department of Traditional Medicine of Tehran University of Medical Sciences, and the Management and the Distinguished Staff. 


\section{بررسى تأثير روغن بهليمو بر اختّلالات خواب سالمندان؛ مطالعه كارآزمايى بالينى دو سو كور}

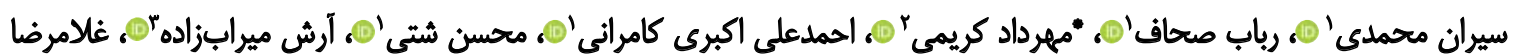

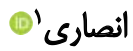

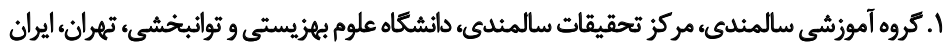

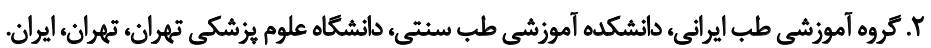

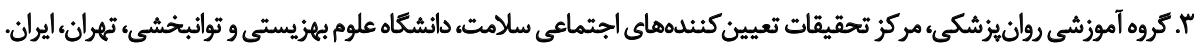

\begin{tabular}{|c|c|}
\hline & 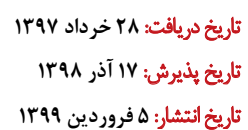 \\
\hline 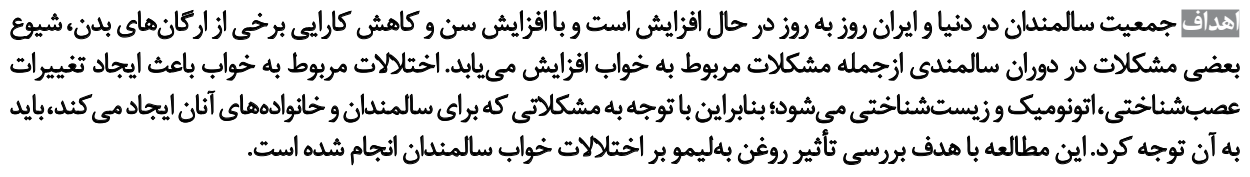 & \\
\hline هواد وروشي هاً يثوهش از نوع كار آزمايى بالينى دو سوكور (stratified design) با كد ثبت IRCT2016061528485N1 و كد اخلاق & \\
\hline IR.USWR.REC.1395.50 در كميته اخلاق دانشكاه علوم بهزيستى و توانبخشى به تصويب رسيده است. در ابتداي مطالعه به صورت & \\
\hline 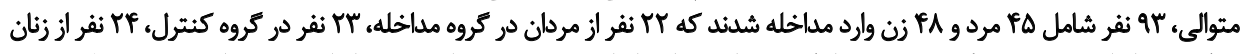 & \\
\hline 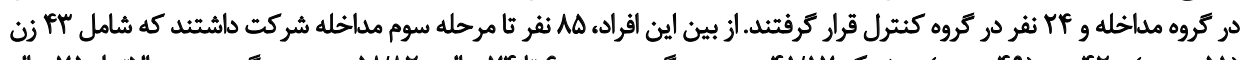 & \\
\hline 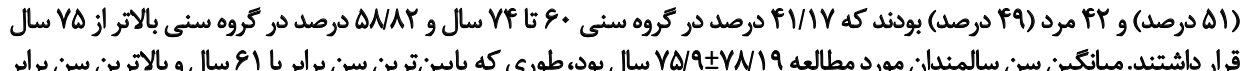 & \\
\hline 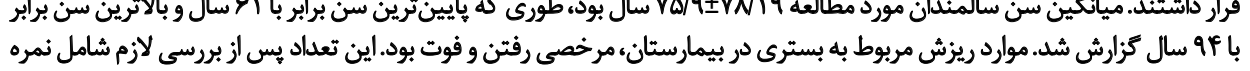 & \\
\hline 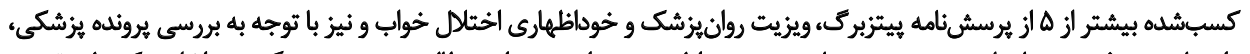 & \\
\hline داروهاى مصرفى و معيارهاى ورود و سيس با توجه به جداول رندوميزاسيون، وارد مطالعه شده و به دو كروه مداخله و كنترل تقسيمي & \\
\hline 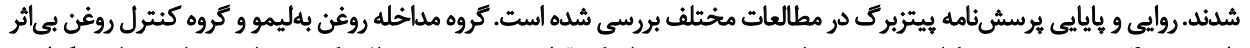 & \\
\hline 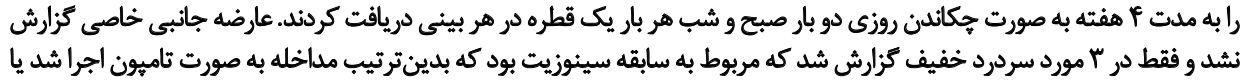 & \\
\hline 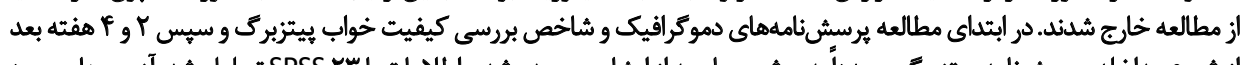 & \\
\hline 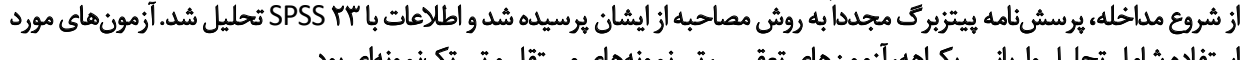 & \\
\hline استفاده شامل تحليل واريائس يكراهل، ازمون هاى تعقيبى، تى نمونههاى مستقل و تى تكنئمونهاى بود. & \\
\hline 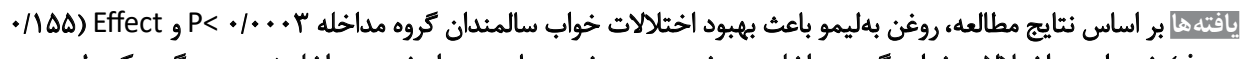 & \\
\hline (size = شده است. اختلالات خواب كروه مداخله در هفته دوم و هفته جهارم بس از شروع مداخله نسبت به كروه كنترل بهبود & كليدوازه内ها: \\
\hline هيافته است. & سالمند، الختتلالات \\
\hline 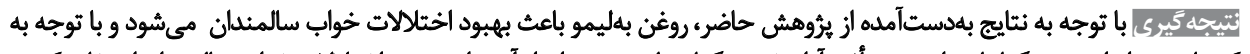 & 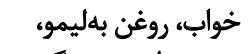 \\
\hline كمعارضه وارزان بودن كياهان دارويى و تأثير آرامبخشى كياه بهليمو مى توان از آن براى بهبود اختلالات خواب سالمئلان استفاده كرد. & يرسش نامه يجيتزبرى \\
\hline
\end{tabular}


تأهل، طبقه اجتماعى، محل سكونت، سلامت جسمى، روانى،

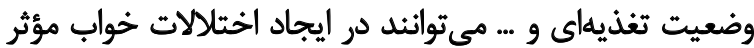

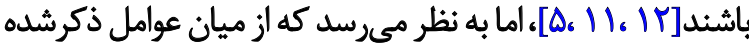

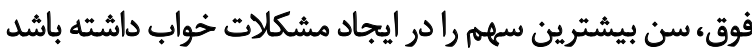

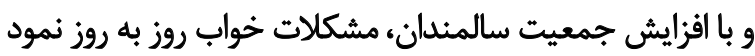

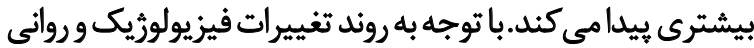

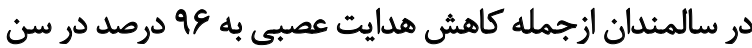

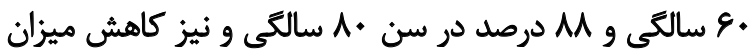

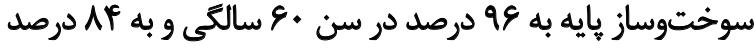

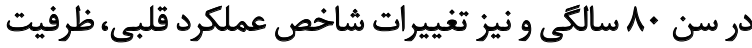

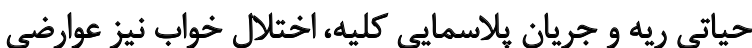

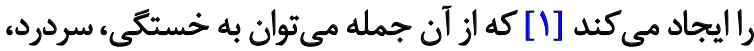

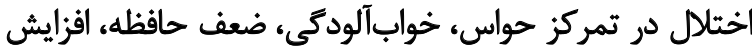

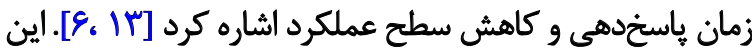

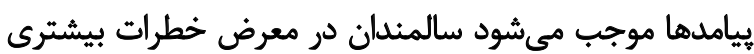

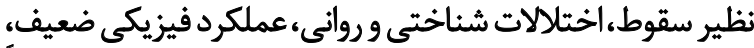

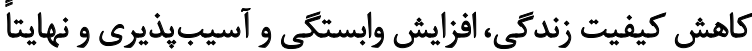

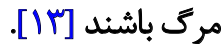

با توجه به تغييرات ذكرشده در دوره سالمندى، بعضى از

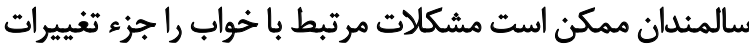
طبيعى مرتبط باسن قلمداد كنيند ود در جستجوى درئ درمان مناسب

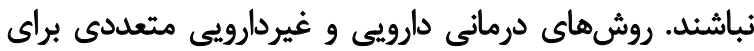

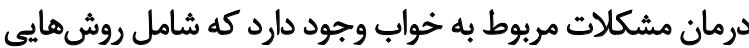

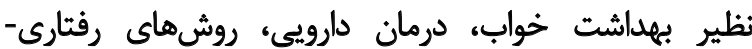

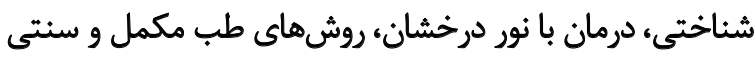

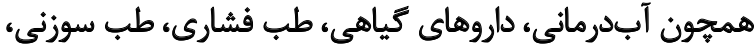

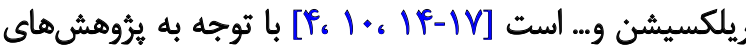

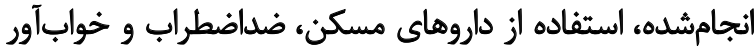

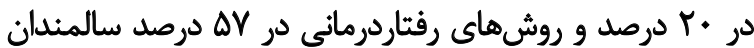

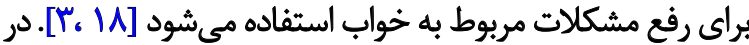

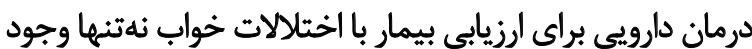

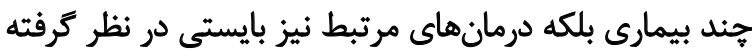

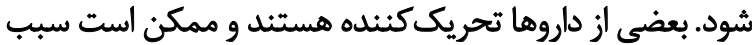

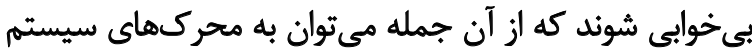

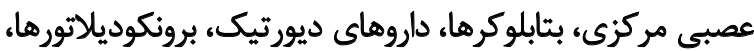
مسدودكنتدهاى دكونزّتانها، ضدافسردىى ها و هورمونهاى تيروئيدى اشاره

كرد [19]

قابل ذكر است كه در سالمندان، دو سوم عوارض ناخواسته

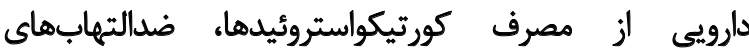
غيراستروئيدى، داروهاى قلبى دارئعروقى و داروهاي مؤثر بر بر رواني

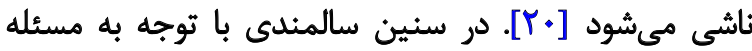

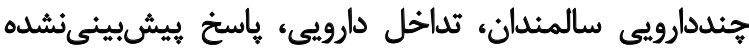

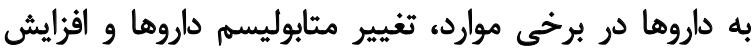

مelad

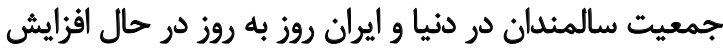

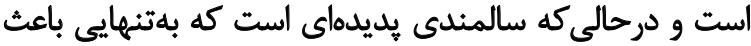

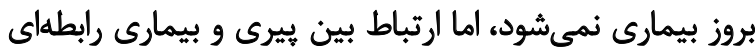

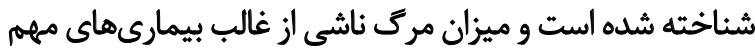

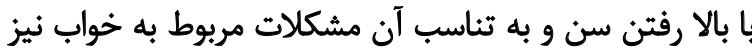

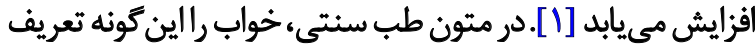

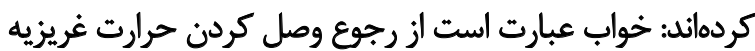

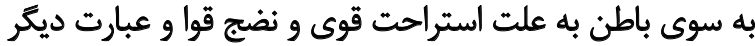

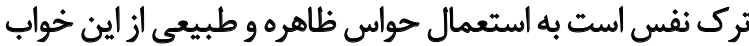

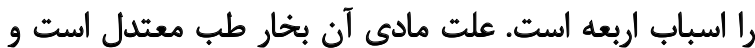

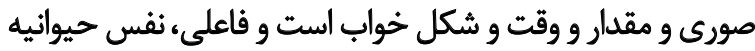

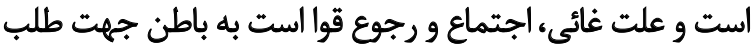

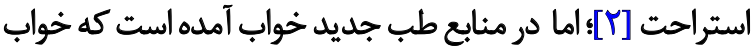

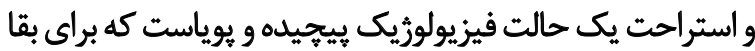

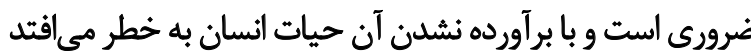

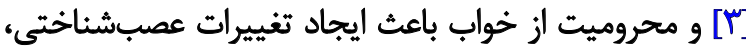

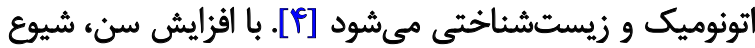

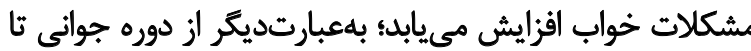

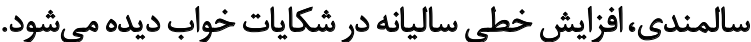

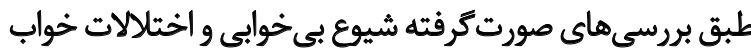

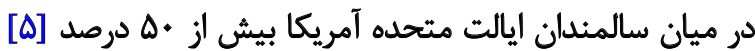

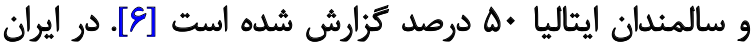

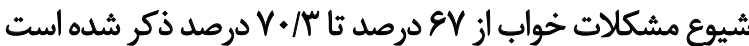

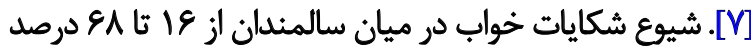

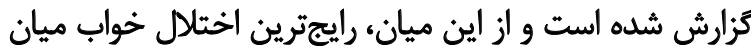

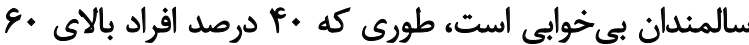

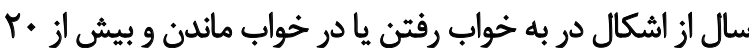

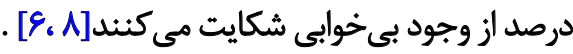
اختلال خواب به نارضايتى از كيفيت مقدار خواب انرزئبشي

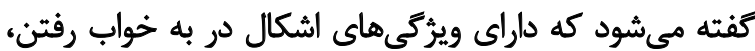

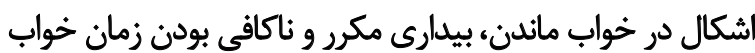

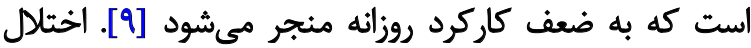

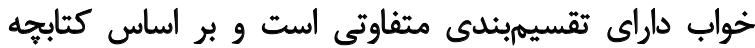

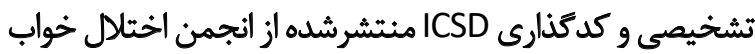

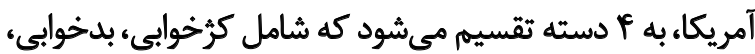

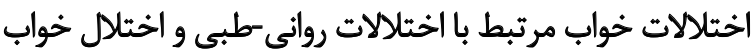

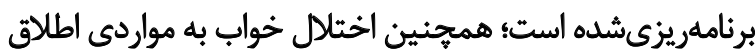

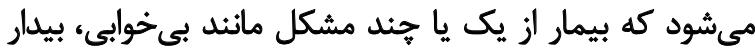

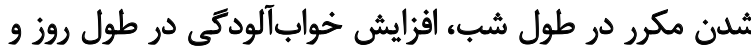

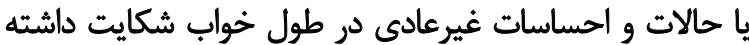

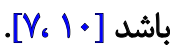

عوامل متعددى نظير جنس، سن، وضعيت اشتغال، وضعيت 
ضداسياسم، كاهنده تب، آرامبخشى و هضم كنيدكى، خاصيت

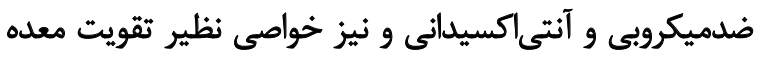

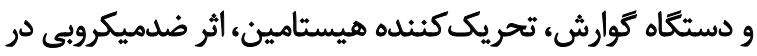

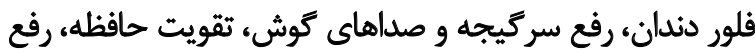

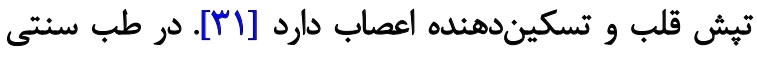

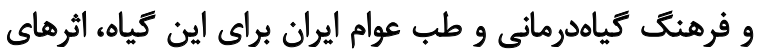

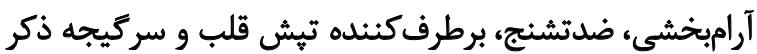

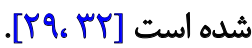

با توجه به تاريخجه طولاني مدت استفاده بومي و محلى ازئ كياه

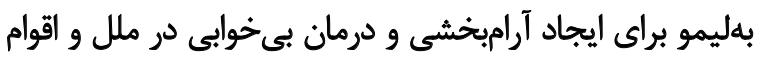

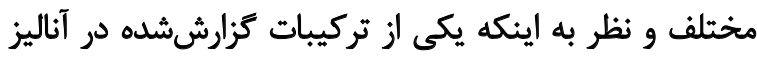

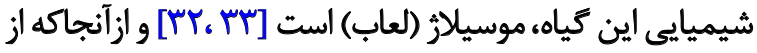

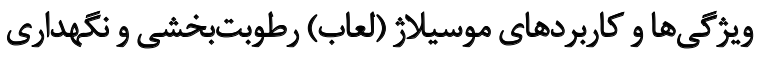

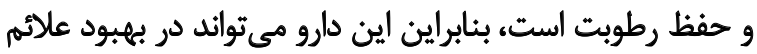

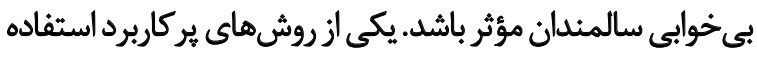

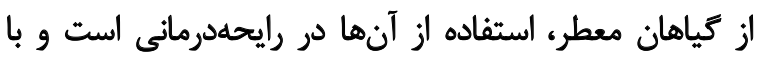

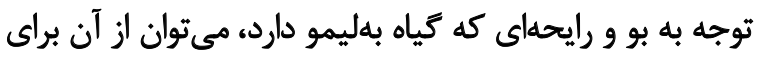

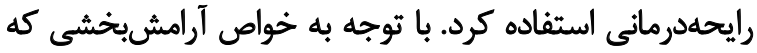

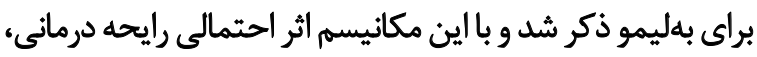

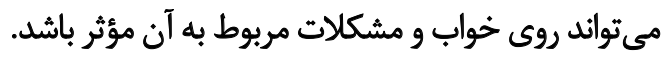

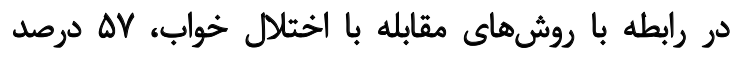

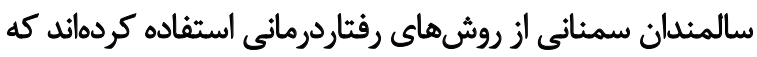

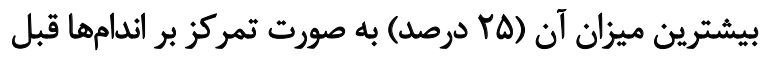

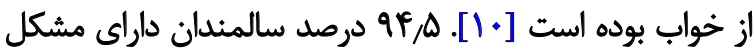

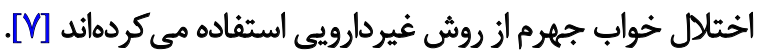

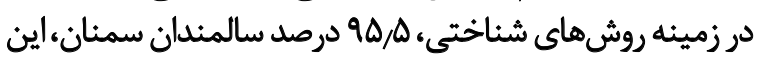

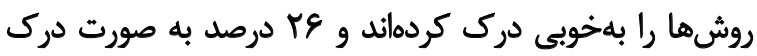
تأثير سن بر خواب بوده است

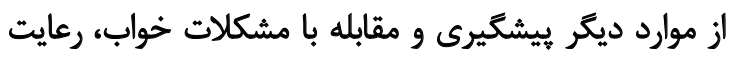

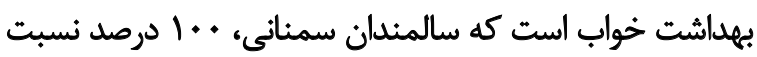

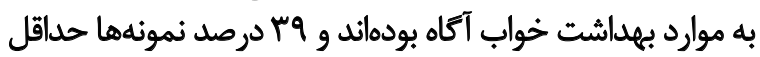
بأ مورد از موارد بهداشتى تأثيركذار بر خواب آشنا بودهاند [ • 1].

مطالعه ساريس و بايرن به صورت مرور سيستماتيك دقيق

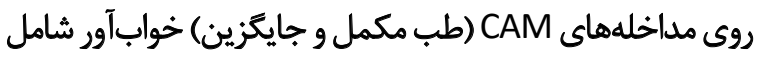

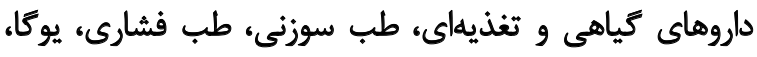

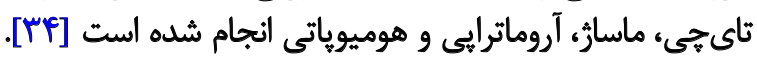
بانكهاى اطلاعاتى الكترونيكى

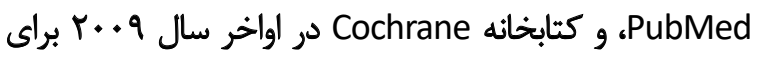

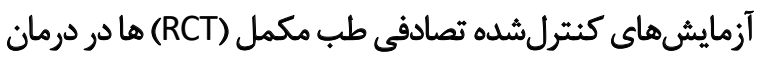

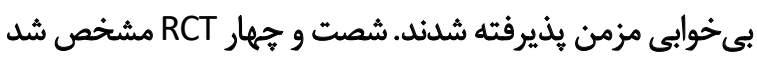

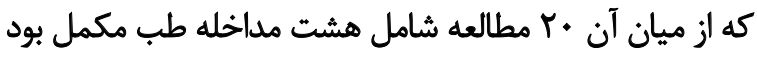

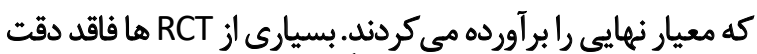

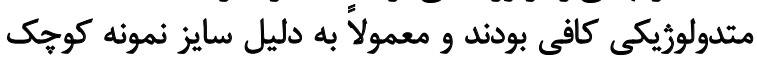

حساسيت به افت عملكرد دستكاه عصبى مركزى و... بايد داروها

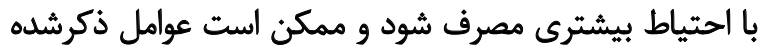

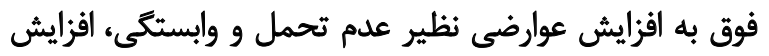

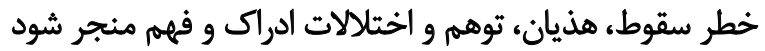

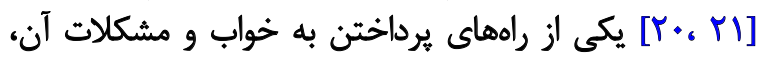

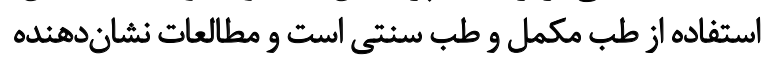

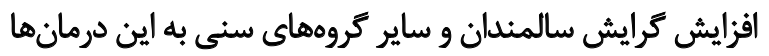

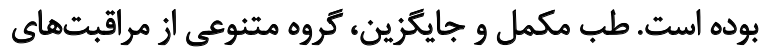

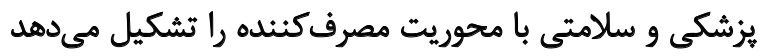

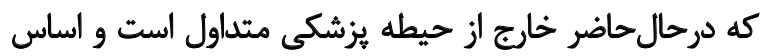

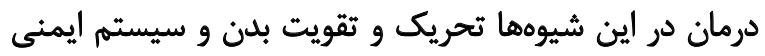

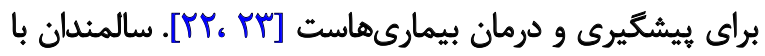

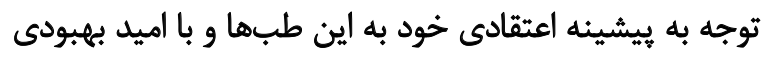

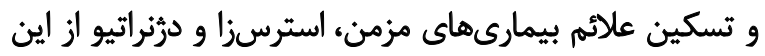

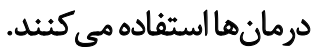

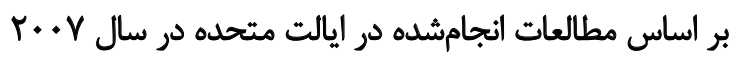

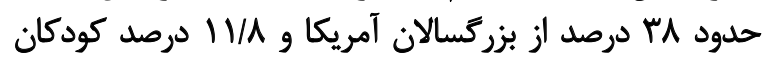

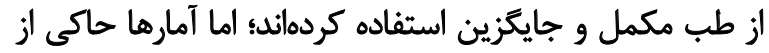

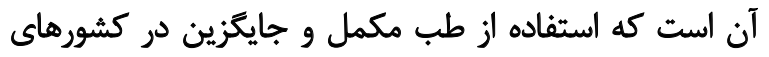

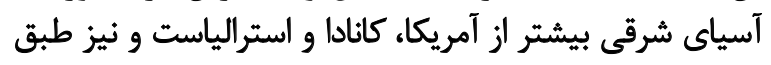

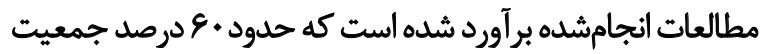

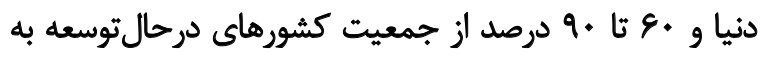

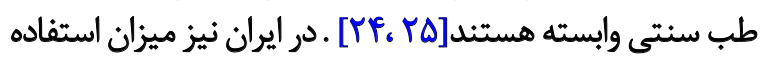

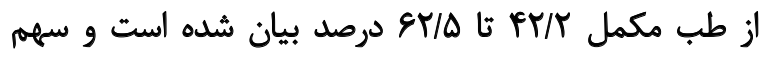

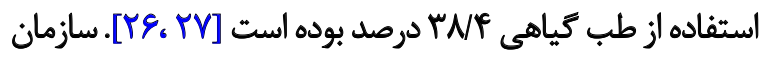

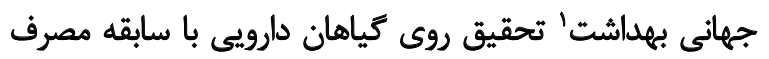

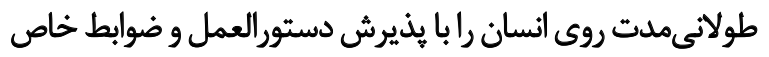

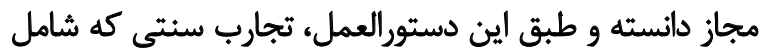

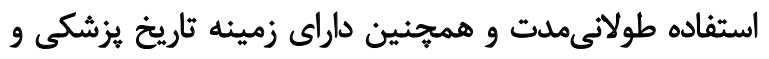

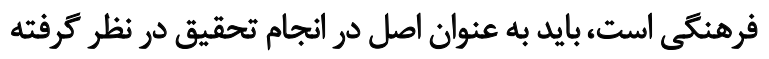

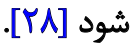

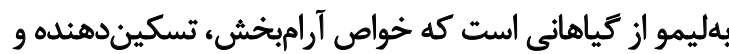

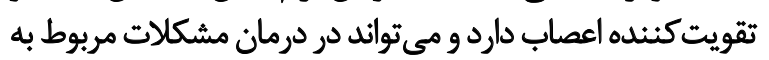

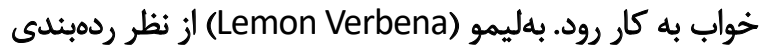

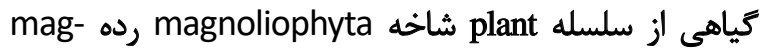

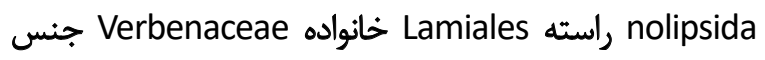

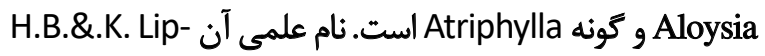

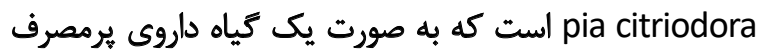

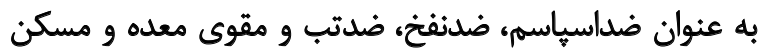

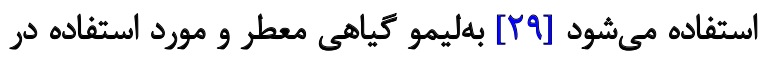

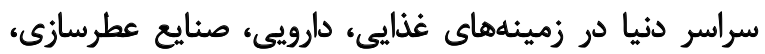

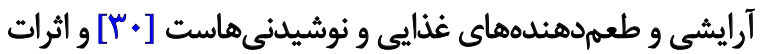

1. World Health Organization 


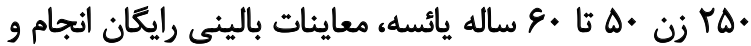

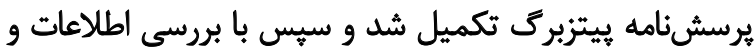

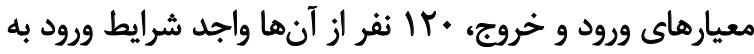

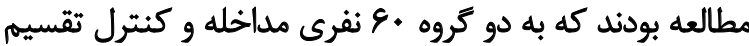

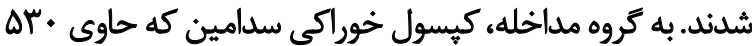

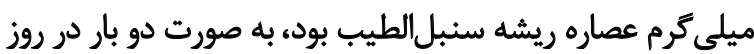

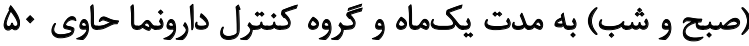
ميلى

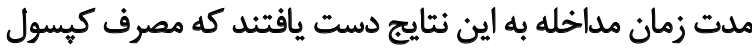

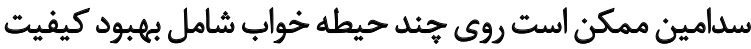

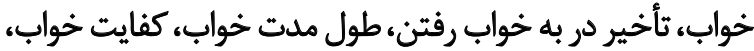

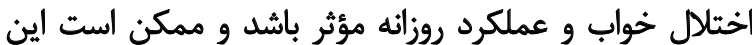

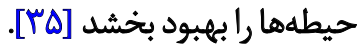

همانكونه كه كفته شد يكى از اثرات ذكرشده در متون كه

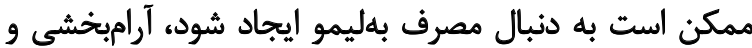

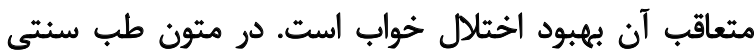

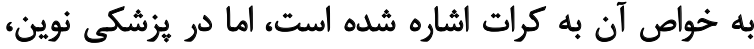

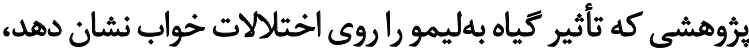

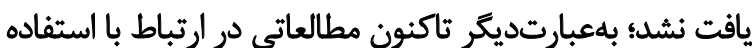

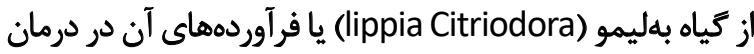

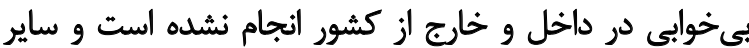

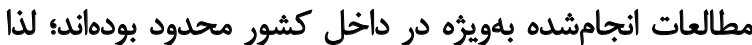

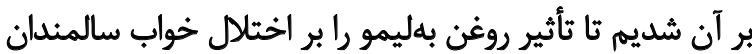

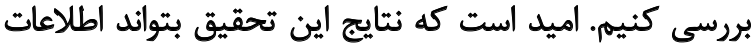

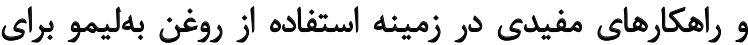

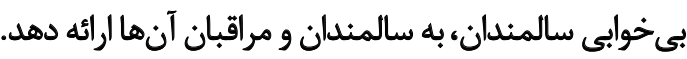

$$
\text { روش مطالعه }
$$

مطالعه از نوع كارآزمايى بالينى شاهددار تصادفىشده دو سو

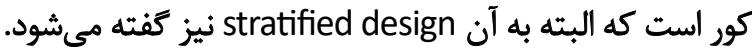

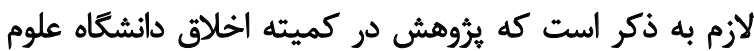

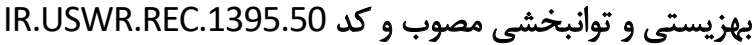

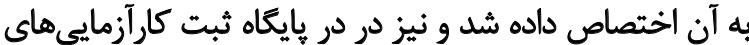

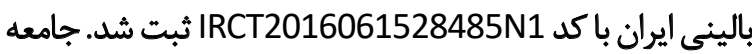

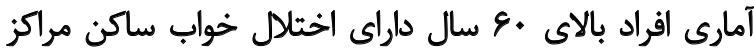

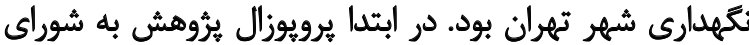

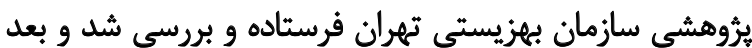

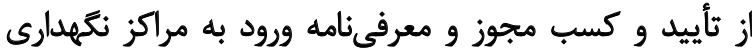

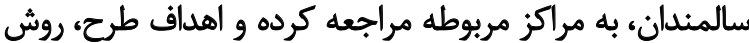

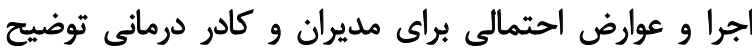

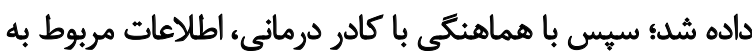

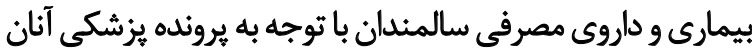

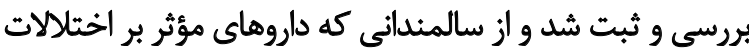

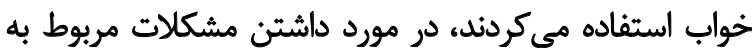

يا شرايط كنترلى ناكافى از بين دادهها حذف مي مشديند. در بين

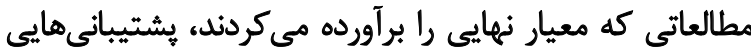

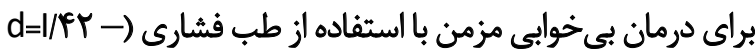

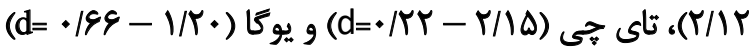

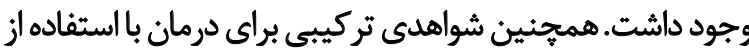

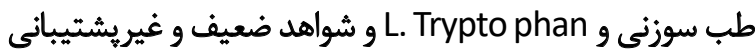

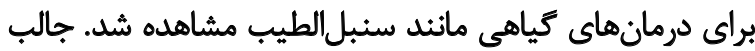

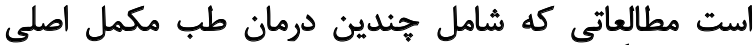

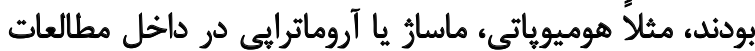

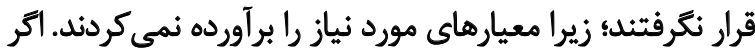

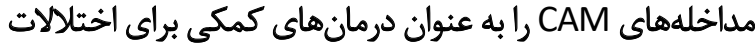

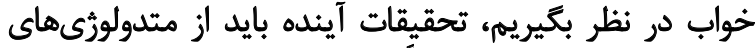

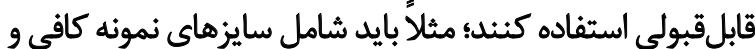

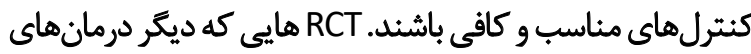
تستنشده CAM ا ارزيابى مى كنثلد، مائند ماسار، هوميوياتى

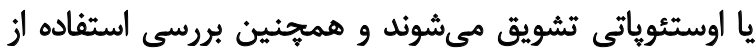

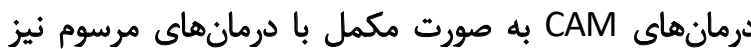

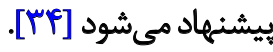

كيمياكر و همكاران در يرؤهشى كه به صورت كار آزمايى بالينى تائى

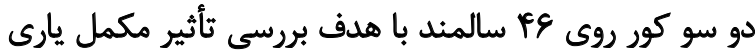

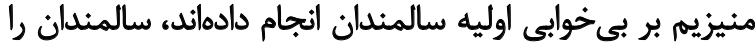

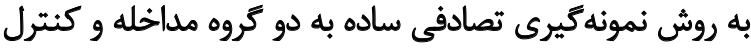

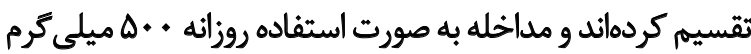

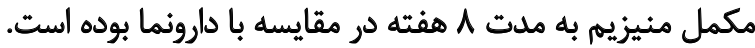

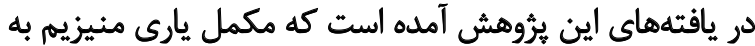

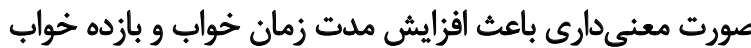

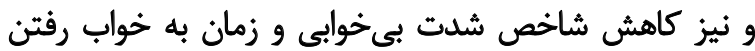

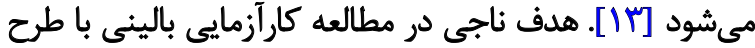

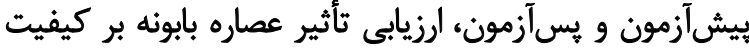

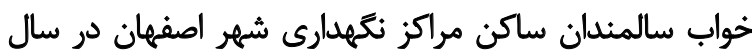

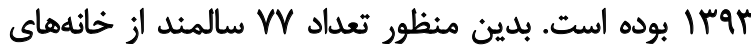
سالمندان با روش نمونه گيرى آسان و مستمر انتخاب و بـ به صورت

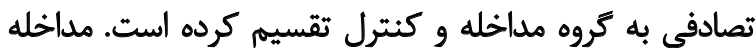

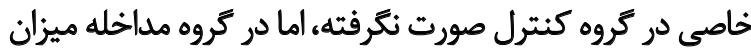
F ميلى F.. .

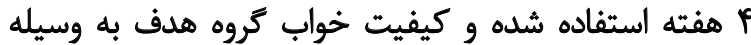

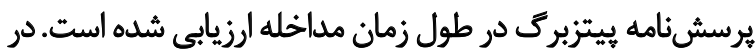

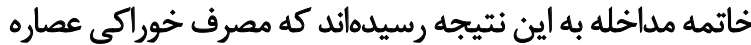

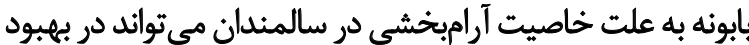

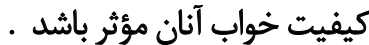

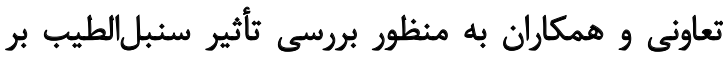

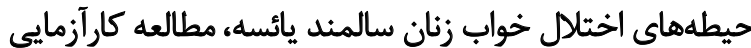

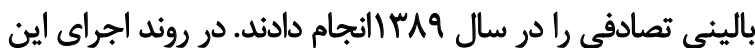

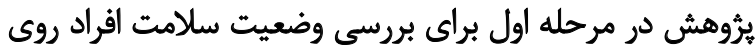


مردان مصرفكنيده دارو و •ع تا Ralance Block: Randomization Table P

Balance Block مردان مصرفكنيده دارو و Va سال به بالا: Randomization Table O

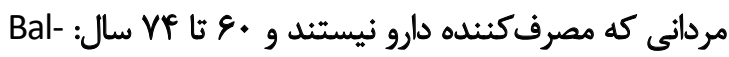
ance Block Randomization Table T

Bal- مردانى كه مصرفكنئده دارو نيستند و VD سال به بالا: ance Block Randomization Table H

زنان مصرفكنيده دارو و •ع تا VF سال: Balance Block Randomization Table S

زنان مصرفكنيده دارو و VA سال به بالا: Balance Block Randomization Table R

زناني كه مصرفكننده دارو نيستند و •ع تال Valance: Block Randomization Table L

زنانى كه مصرفكننده دارو نيستند و VD سال به بالا: Bal ance Block Randomization Table M

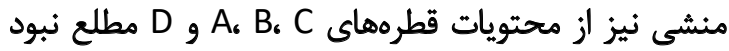

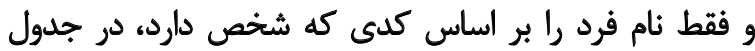

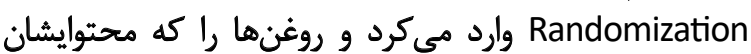

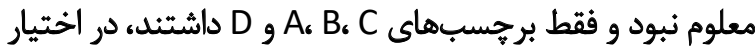

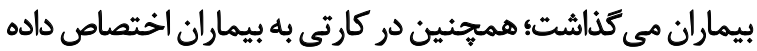

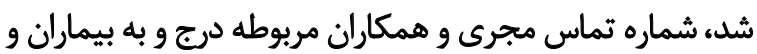

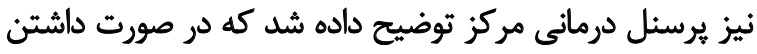

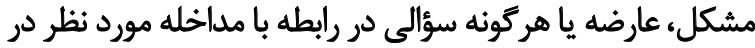

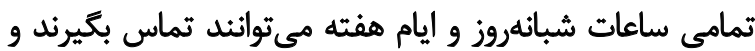
مسئله مورد نظر راكزارش كنئد

همان كونه كه ذكر شد، بيمار ان با توجه به جدول رون رئدوميزاسيون

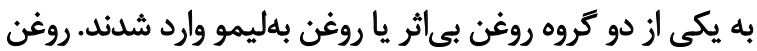

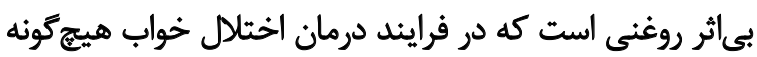

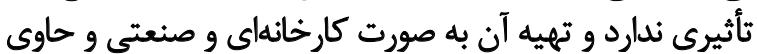

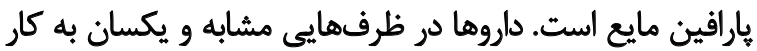

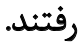

فرايند تهيه روغن بهليمو: •له كرم نمونه تهيهشده و مورد

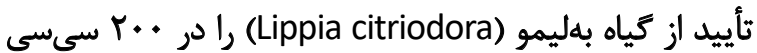

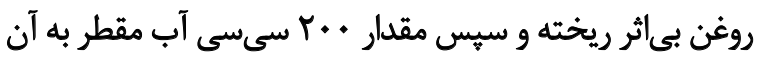

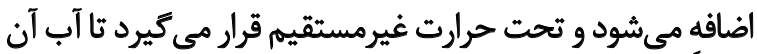

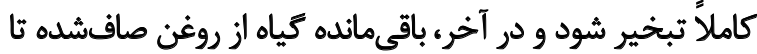

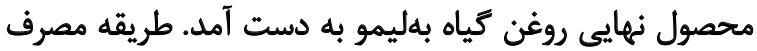

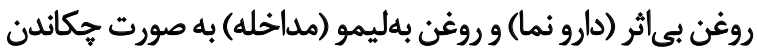
يك قطره از روغن مربوطه در صبح و شب بوده است.
خواب سؤال يرسيده شد؛ همجنين يزشك و روانيزشك آنها

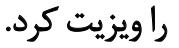

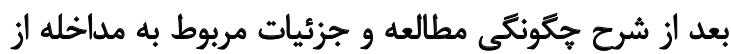

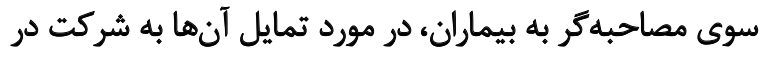

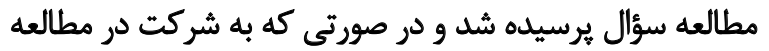

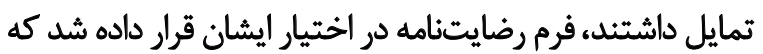

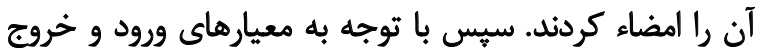

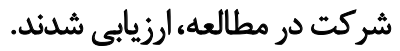

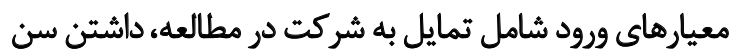

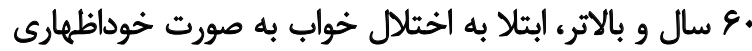

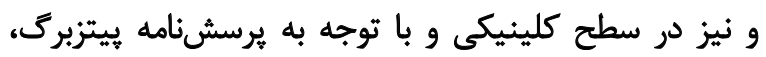

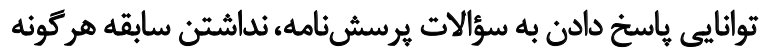

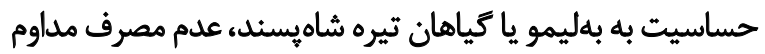

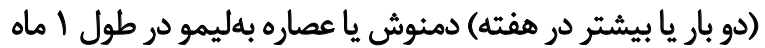

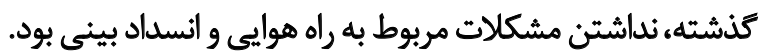

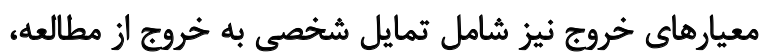

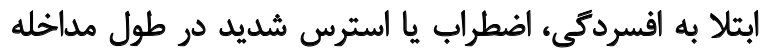

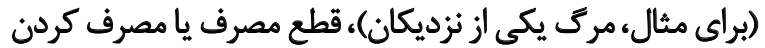

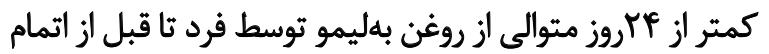

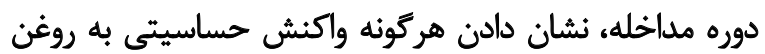

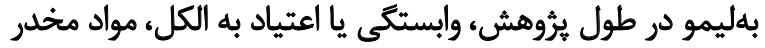

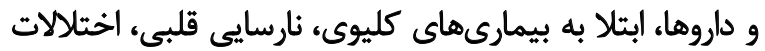

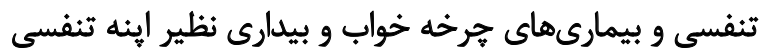

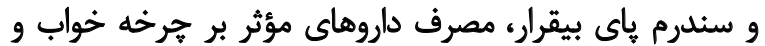

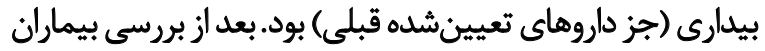

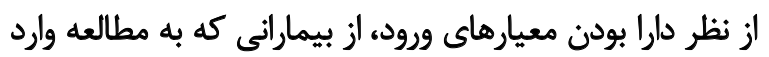

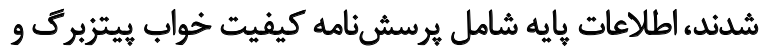
اطلاعات دموكرافيك يرسيده شد.

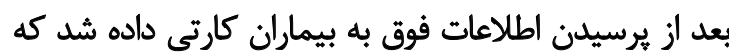

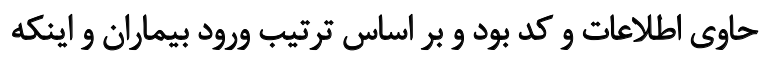
به كدام جدول balanced - block randomization وارد وارد

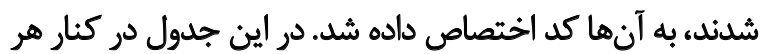

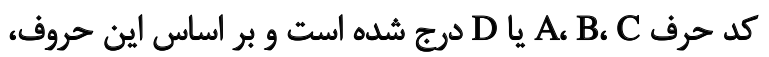

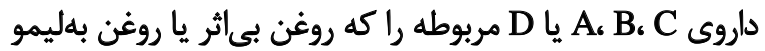

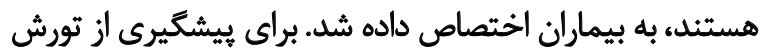

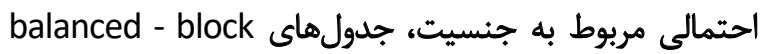

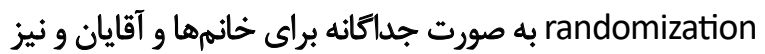

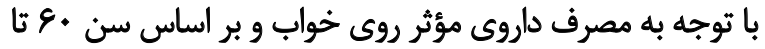

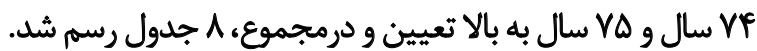

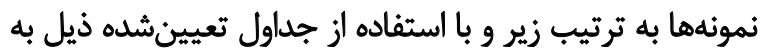
كروههاى مداخله و كنترل اختصاص داده شدئند 
بيانكر وجود وضعيت طبيعى، مشكل خفيف، متوسط و شديد

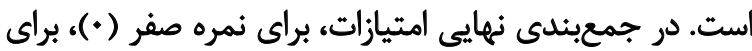

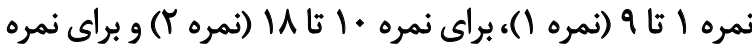

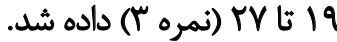

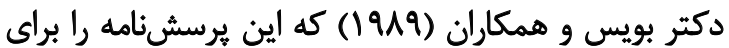

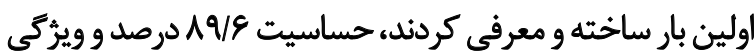

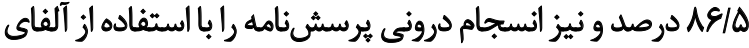

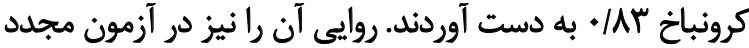

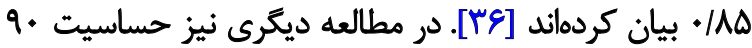

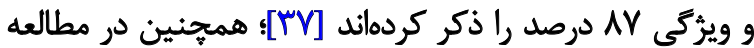

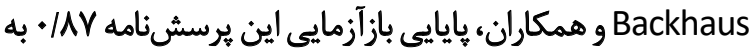

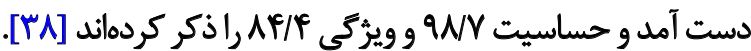

Andrykowski , Carpener ا براى اين مقياس به دست آوردند و

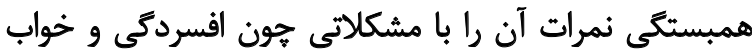

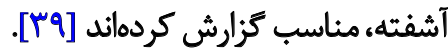

بايايي يرسشنامه در مطالعهاى كه داداشيور و همكارانش

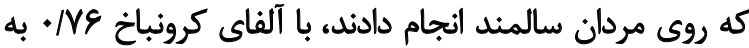

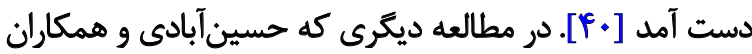

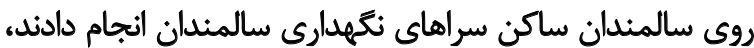

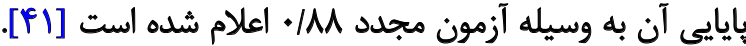
يرسشنامه دموكرافيك رانيز اساتيد ثأييد كردند.

لازم به ذكر است كه در تمام مراحل يُروهش خود را ملزم

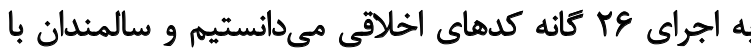

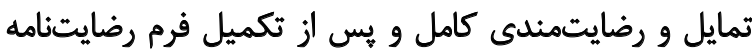

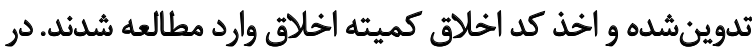
ابتداى شروع مداخله و بعد از ورود افر اد بله مطالعها، روش كار كار برائ

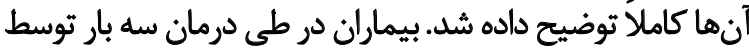

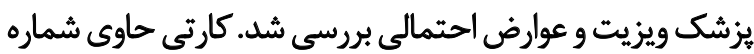

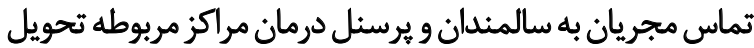

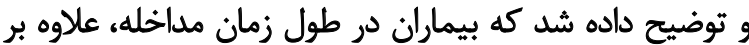

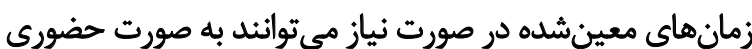

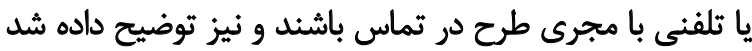

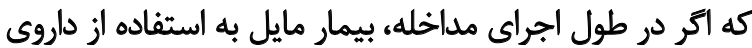

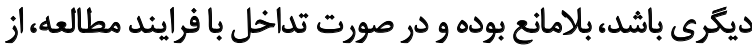

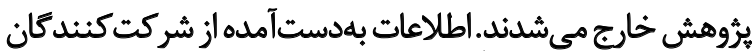

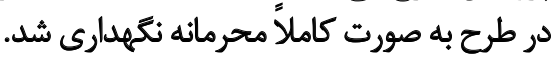

يافتهلها

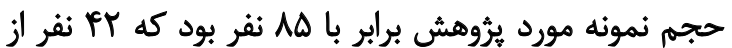

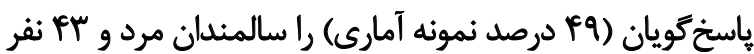
از ياسخ كويان (اله درصد نمونه آمارى) را سالمئدان زن تشكيل

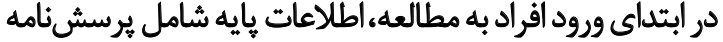

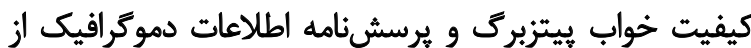

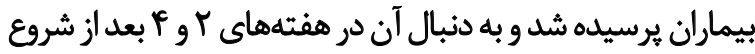

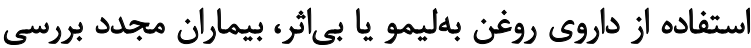

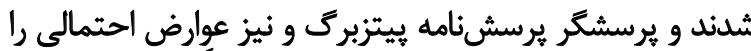

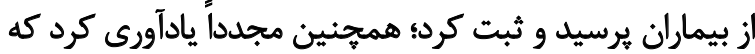

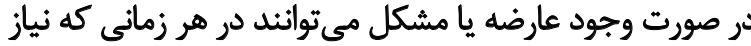

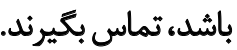

لازم به يادآورى است كه بيمار و مصاحبه كنينده هر دو از دارو يا

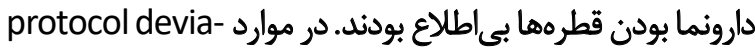

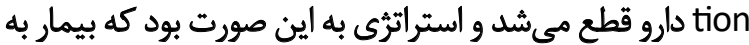

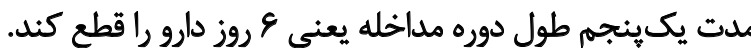

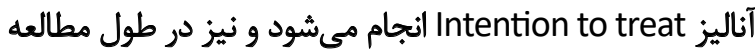
براى كنترل و بيخيرى مصرف صحيح داروهاو ساير موارد احتمالى

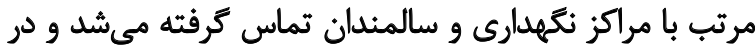

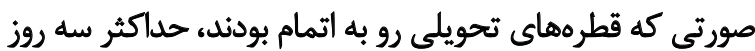

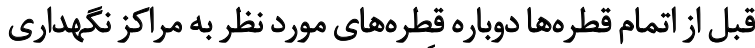

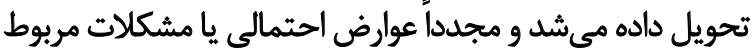

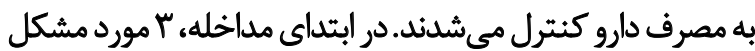

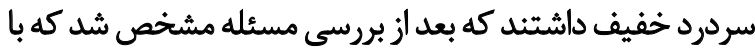

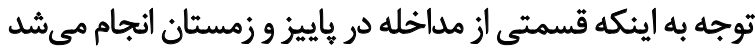

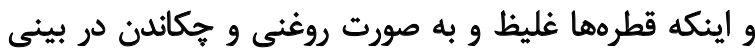

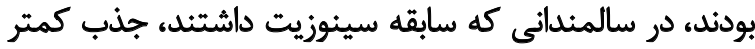

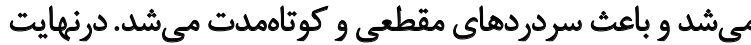

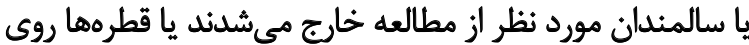

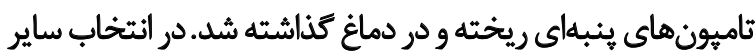

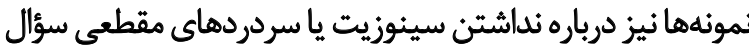
مىشد كه در صورت داشتن اين موارد به مداخله وارد نمى نشئنداند.

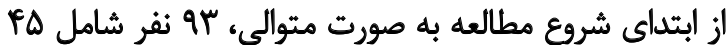

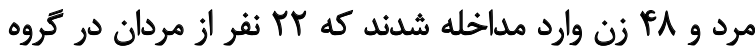

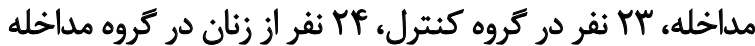

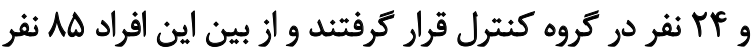

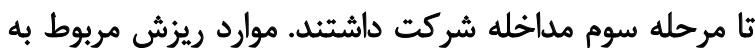
بسترى در بيمارستان، مرخصى رفتن و فوت داخت بودي مورد ريز

يرسشنامه كيفيت خواب بيتزبورى از اعتبار علمى مناسب

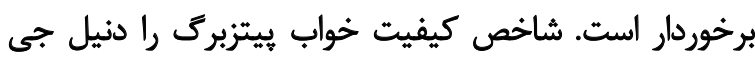

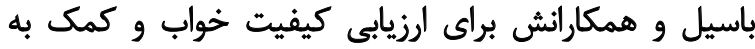

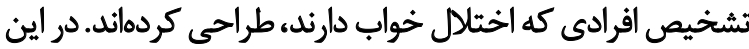

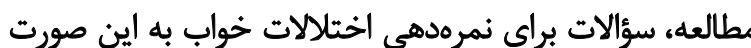

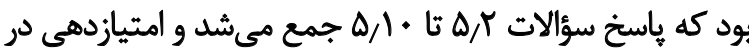

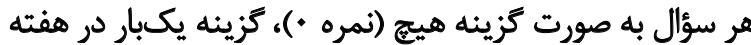

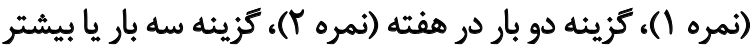

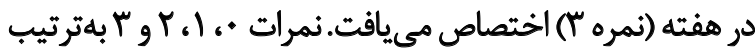


جدول ا. آمارههاى توصيفى مربوط به شاخص اختيلالات خواب در سه مرحله نموئهكيرى

\begin{tabular}{|c|c|c|c|}
\hline ت تعداد & مياتكين +انحراف معيار & توره & 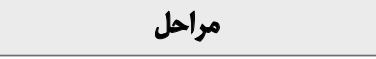 \\
\hline$m$ & $11 / \cdot 9 \pm m / m$ & كروه كنترل & \\
\hline r. & سח/\% & كروه مداخله & اختلالات خواب قبل از ملاخله \\
\hline$v^{e}$ & $11 / \cdot \Delta \pm T / q r$ & كل & \\
\hline$M$ & $1.18 \Delta \pm F / 94$ & كروه كنترل & \\
\hline$r+$ & NIV \pm M/RY & كروه مداخله & اختلالات خواب دو هفته يس أز مداخله \\
\hline$n$ & $\checkmark / \mu \pm F / M$ & كل & \\
\hline 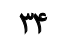 & $\| r / M \mid \pm r / 4$ & كروه كنترل & \\
\hline r. & $q r \cdot \pm F / r$ & كروه مداخله & اختلالات خواب يك ماه بعد از مداخله \\
\hline$n^{e}$ & $1 \cdot(A) \pm f / \pi V$ & كل & \\
\hline
\end{tabular}

il

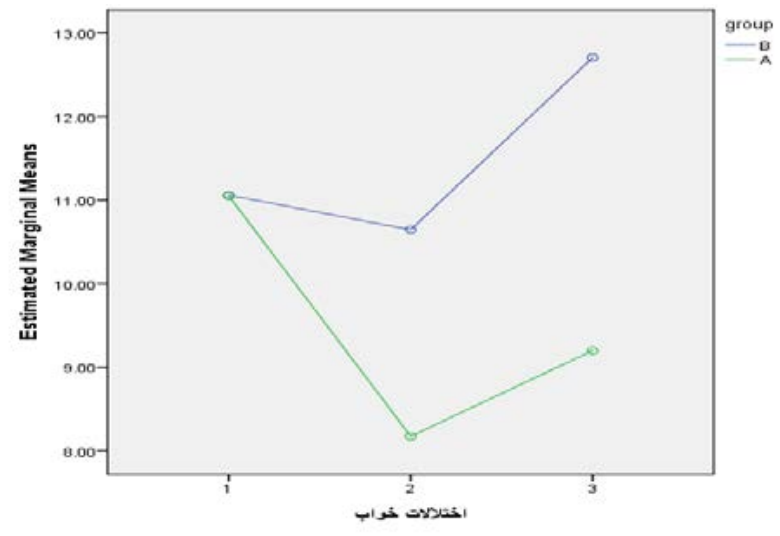

配

از نظر وجود بيمارىهاي مزمن از مجموع سالمندان ياسخكو در

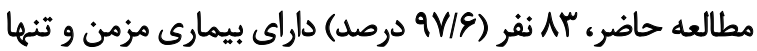

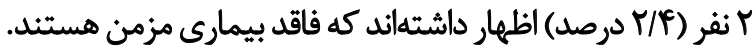

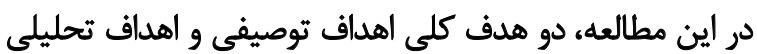

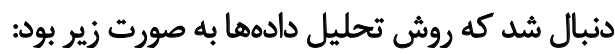
در اهداف توصيفى براي متغيرهاي ياسخ مورد مطالعه كه

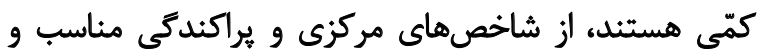
براي متغيرهاي كيفى از توزيع فراوانى و براي متغير هائ كتئي

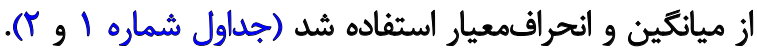

تصوير ا. نمودار mean plot اختلالات خواب بر حسب دو كروه

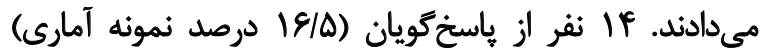

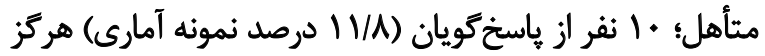

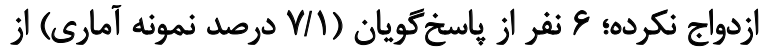

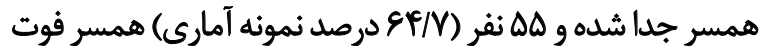

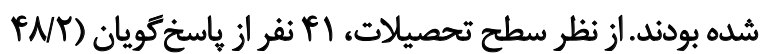

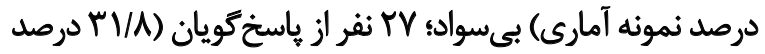

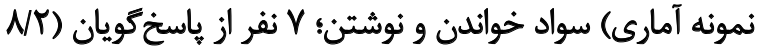

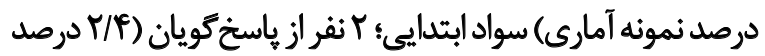

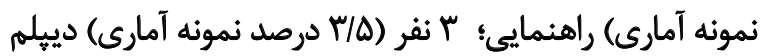

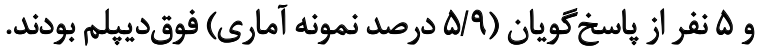

جدول r. مقايسه ميانكينين نمره اختلالات خواب بين كووه مداخله و كروه شاهد در طول زمان مداخله

\begin{tabular}{|c|c|c|c|c|c|c|c|}
\hline \multirow{2}{*}{$\begin{array}{c}\text { Effect } \\
\text { Size }\end{array}$} & \multirow{2}{*}{$\mathbf{P}$} & \multirow{2}{*}{ Fدم F F } & \multicolumn{3}{|c|}{ ميانكيند|انحراف معيار } & \multirow{2}{*}{ ت تروه } & \multirow{2}{*}{ 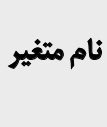 } \\
\hline & & & يك ماه بس از شروع مداخله & دو هفته يس ازثشروع مداخله & قبل از شروع مداخله & & \\
\hline \multirow{2}{*}{.$/ 1 \Delta \Delta$} & \multirow{2}{*}{$.10 .+4$} & VNEINET & $f / r i \pm q / T$. & $r / T T \pm N I V$ & $\varphi / \pi+11 / \cdot \Delta$ & ملخاخله & اختلالات \\
\hline & & V/lier & $r / 4 \cdot \pm \mid r / M$ & $r / 8 Y \pm 1 \cdot 180$ & $m / f f \pm 11 / .8$ & 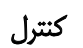 & خواب \\
\hline
\end{tabular}


نسبت به گروه كنترل كاهش يافت و بهعبارت ديكر روغن بهليمو روى اختلالات خواب سالمندان مؤثر بود.

تأثير آروماترايى با اسانس اسطوخودوس بران بران كيفيت خواب

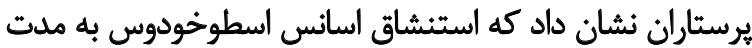

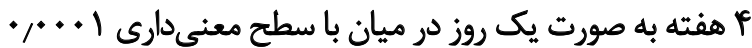

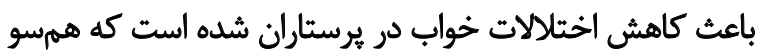

با بتايج مطالعه حاضر بوده است [Fr]

نصيرى و همكارانش دريافتند كه بازتابدرمانى بر اختلالات

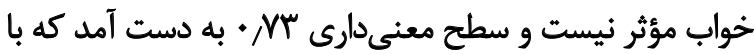

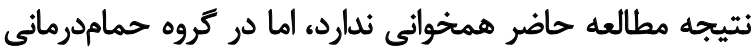

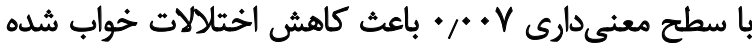

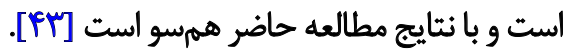

مصرف كيسول سدامين به مدت يكماه به صورت دو دو بار در روز

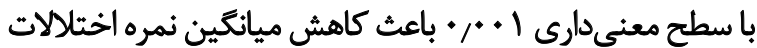
خواب زنان يائسه شده و مؤثر بوده است كه باعث بانتايج مطالعه ماضين نمره

همخوانى دارد [FF]

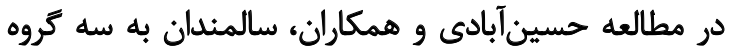

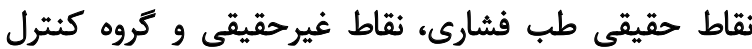

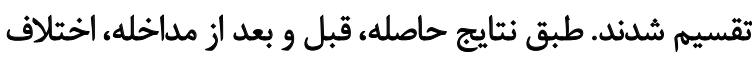

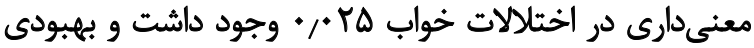

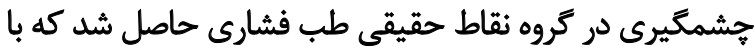

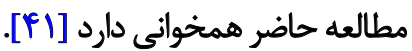

باتوجه به اينكه تمامى مطالعات انساني و مخصوصاً يُوهشي هاي

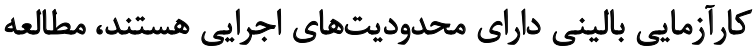

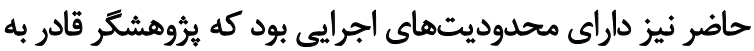

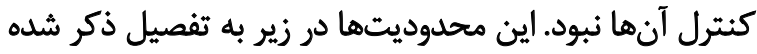

با توجه به جستجوهاي انجامشده و بررسى منابع و مطالعات

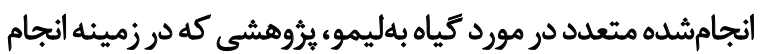

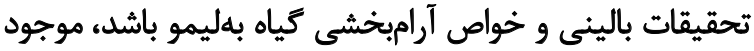

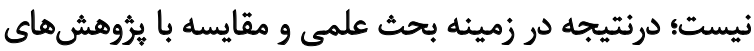

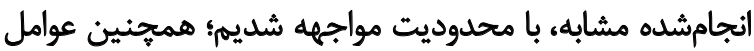

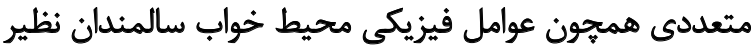

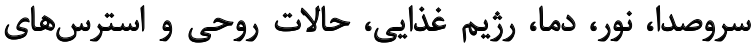

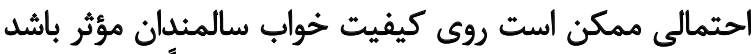

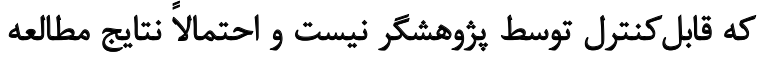

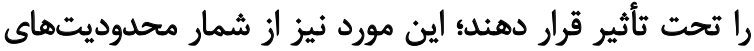

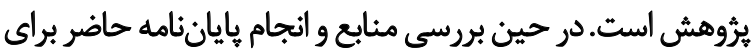

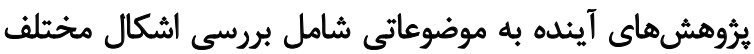

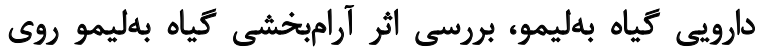

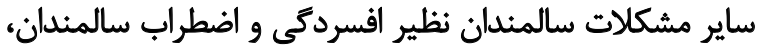
مطالعاتى براى تعيين دوز و مكانيسم اثر كياه بهليمو، مقايسه
در مواردى نيز براى توصيف يراكندگى از حداقل و حداكثر و

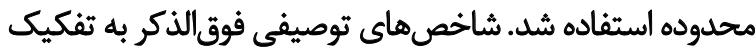

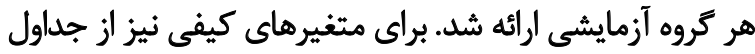

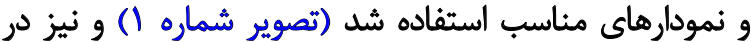

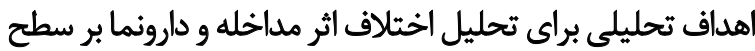

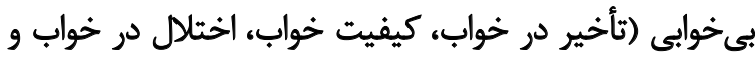

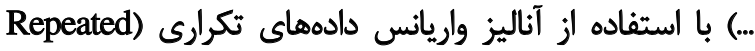
Measure Analysis of Variance

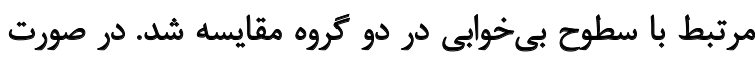

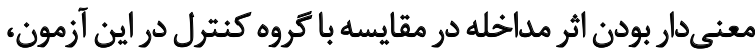

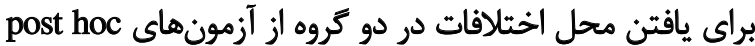
مناسيس ايستئفاده شُّ.

ses اين يُروهش با هدف بررسى تأثير روغن بلهليمو در مقايسه

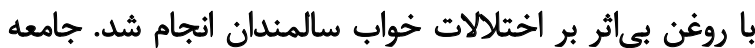

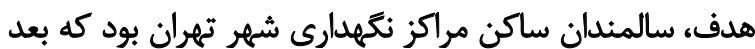

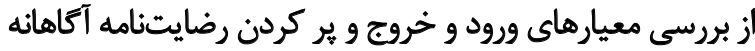

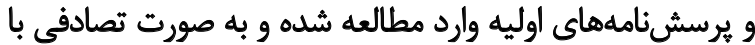

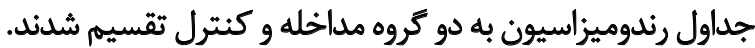

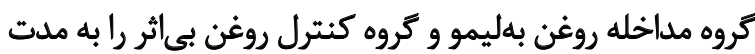

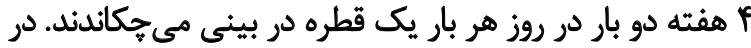

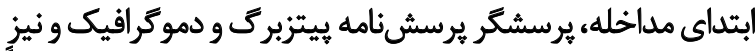

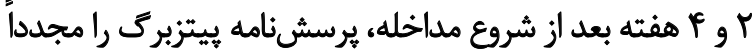

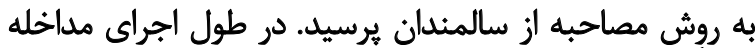

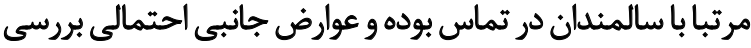

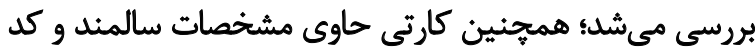

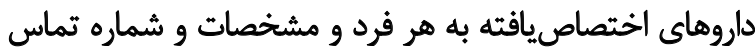

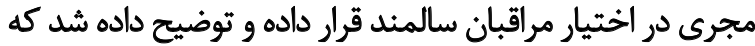

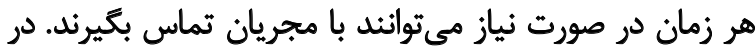

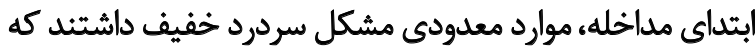

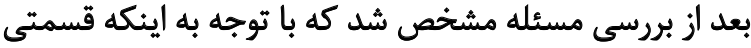

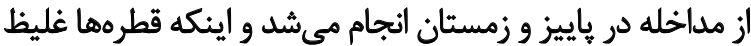

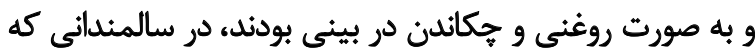

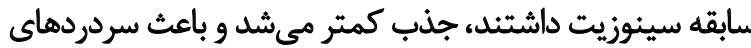

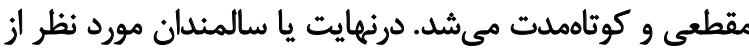

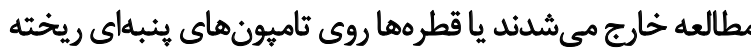

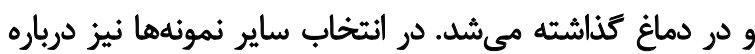

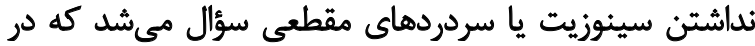
صورت داشتن اين موارد به مداخله وارد نمى شُدنديند. فرضيه مورد بررسى اينگونه بود كه ميانكين نمره اختلالات

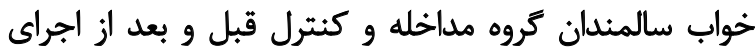

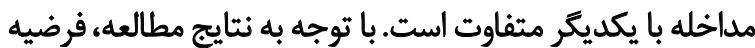

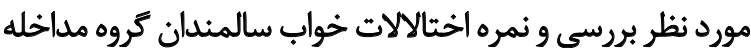




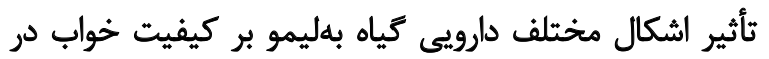

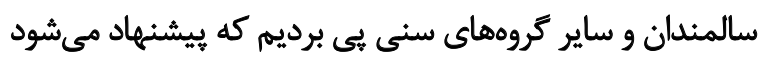

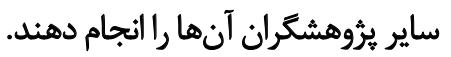

\section{نتيجلكَيرى نُهايى}

بر اساس نتايج بهدست آمده از يثوهش حاضر، استفاده از روغن

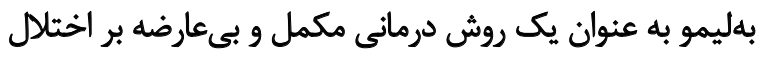

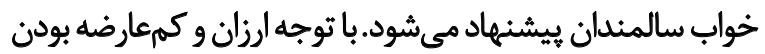

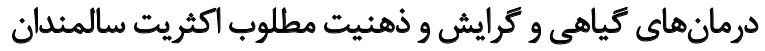

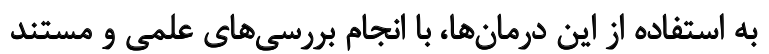

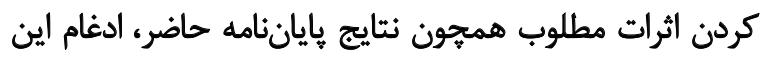

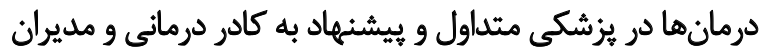

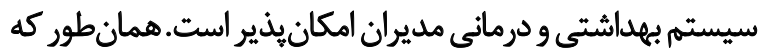

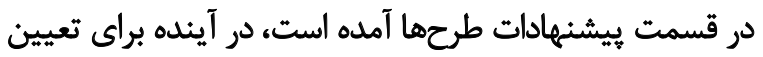

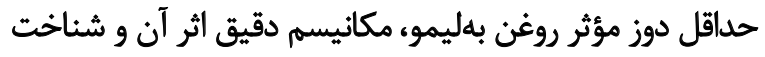

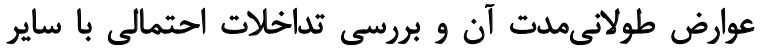

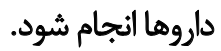

مالاحظات اخلاقي

بيروى أز اصول الخلاق يُوهش

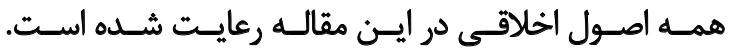

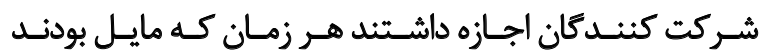

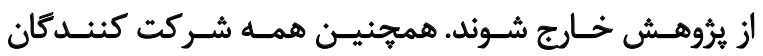

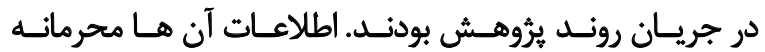

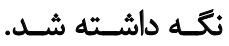

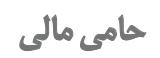

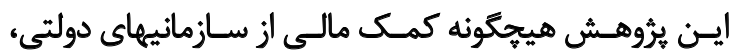

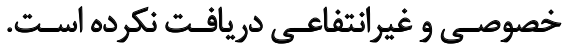
مشاركت نويسندكَّان

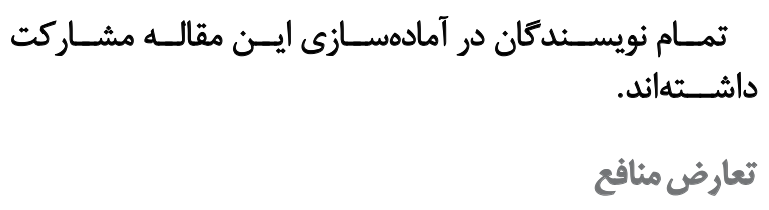
بنابر اظهار نويسندكان، اين مقاله تعارض منافع ندارد.

$$
\text { تشكر وقدروانى }
$$

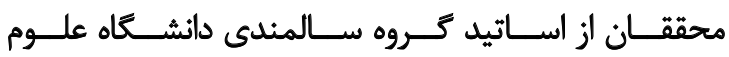

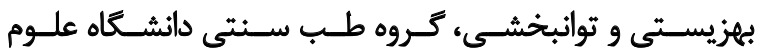

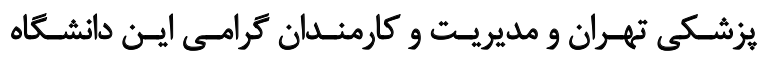
قدردانسـى مى كنئــــد. 


\section{References}

[1] Spence AP. Biology of human aging. Tehran: Publication of University of Welfare and Rehabilitation Sciences; 1929. p. 354.

[2] Gilani MK. Hefzo lseha Naseri. $2^{\text {th }}$, editor. Tehran: Iranian Traditional Medicine Publications; 2015. p. 309.

[3] Hazzard WR, Blass JP, Halter JB, Ouslander JG, Tinetti ME. Principles of geriatric medicine and gerontology.New York: McGrawHill; 2003.

[4] Ham RJ. Primary care geriatrics: A case-based approach. Amsterdam, Netherlands: Elsevier Health Sciences; 2007.

[5] Jung K-I, Song C-H, Ancoli-Israel S, Barrett-Connor E. Gender differences in nighttime sleep and daytime napping as predictors of mortality in older adults: the Rancho Bernardo study. Sleep medicine. 2013; 14(1):12-9. [DOI:10.1016/j.sleep.2012.06.004] [PMID] [PMCID]

[6] Marasinghe C. Sleep disorders in elderly. Journal of the Ceylon College of Physicians. 2014; 43(1-2). [DOI:10.4038/jccp. v43i1-2.6815]

[7] Torabi S, Shahriari L, Zahedi R, Rahmanian S, Rahmanian K. A survey the prevalence of sleep disorders and their management in the elderly in Jahrom City, 2008. Journal of Jahrom University of Medical Sciences. 2013; 10(4):31. [DOI:10.29252/jmj.10.4.35]

[8] Grewal RG, Doghramji K. Epidemiology of insomnia. Clinical handbook of insomnia. Berlin, Germany: Springer; 2017. pp. 1325. [DOI:10.1007/978-3-319-41400-3_2]

[9] Ohayon MM. Epidemiology of insomnia: what we know and what we still need to learn. Sleep medicine reviews. 2002; 6(2):97111. [DOI:10.1053/smrv.2002.0186] [PMID]

[10] Nobahar M, Vafai AA. Assessment of Elderlies Sleep Disorders and Different Confronts Methods Among Them. Iranian Journal of Ageing. 2007; 2(2):263-8.

[11] Arasteh M, Yousefi F, Sharifi Z. Investigation of sleep quality and its influencing factors in patients admitted to the gynecology and general surgery of besat hospital in sanandaj. Medical Journal of Mashhad University of Medical Sciences. 2014; 57(6):762-9.

[12] Khalili Z, Sadrollahi A, Aseman E, Gholipour F, Abbasian M. Evaluation factors associated with sleep quality in the elderly shahid Beheshti hospital of Kashan. Journal of Geratric Nursing. 2016; 2(3):32-43.

[13] Abbasi B, Kimiagar M, Sadeghniiat K, Shirazi MM, Hedayati $\mathrm{M}$, Rashidkhani B. The effect of magnesium supplementation on primary insomnia in elderly: A double-blind placebo-controlled clinical trial. Journal of research in medical sciences: the official journal of Isfahan University of Medical Sciences. 2012; 17(12):1161.

[14] Gooneratne NS. Complementary and alternative medicine for sleep disturbances in older adults. Clinics in Geriatric Medicine. 2008; 24(1):121-38. [DOI:10.1016/j.cger.2007.08.002] [PMID] [PMCID]

[15] Ahmadi S, Khankeh H, Mohammadi F, Khoshknab F, Reza Soltani $\mathrm{P}$. The effect of sleep restriction treatment on quality of sleep in the elders. Iranian Journal of Ageing. 2010; 5(2). http:/ / salmandj.uswr.ac.ir/article-1-282-en.html

[16] Akhondzadeh S, Daliri-hampa A. Herbal medicine in sleep disorders. Journal of Medicinal Plants. 2004; 1(9):75-84.
[17] Arab Z, Shariati A, Bahrami H, Asayesh H, Vakili M. The effect of acupressure on quality of sleep in hemodialysis patients. Journal of Urmia Nursing And Midwifery Faculty. 2012; 10(2). http:/ unmf.umsu.ac.ir $/$ browse.php?a_id=828\&sid=1\&slc_lang=en

[18] Abdullahzadeh M, Naji S. The effect of matricaria chamomilla on sleep quality of elderly people admitted to nursing homes. Iran Journal of Nursing. 2014; 27(89):69-79. [DOI:10.29252/ijn.27.89.69]

[19] Sasai T, Inoue Y, Komada Y, Nomura T, Matsuura M, Matsushima E. Effects of insomnia and sleep medication on health-related quality of life. Sleep medicine. 2010; 11(5):452-7. [DOI:10.1016/j. sleep.2009.09.011] [PMID]

[20] Saboor M. Elderly's Medical Therapy Status. Iranian Journal of Ageing. 2007; 2(1):216-22.

[21] Leger D, Poursain B, Neubauer D, Uchiyama M. An international survey of sleeping problems in the general population. Current Medical Research and Opinion. 2008; 24(1):307-17. [DOI:10.1185/030079907X253771] [PMID]

[22] Jafari S, Abdollahi M, Saeidnia S. Personalized medicine: A confluence of traditional and contemporary medicine. Alternative Therapies in Health \& Medicine. 2014; 20(5):31-40. https:// pubmed.ncbi.nlm.nih.gov/25141369/

[23] Mousavizadeh K, Ansari H. Complementary/alternative medicine and medical education. 2008; 7(4): 329-36.

[24] Molassiotis A, Fernadez-Ortega P, Pud D, Ozden G, Scott JA, Panteli V, et al. Use of complementary and alternative medicine in cancer patients: a European survey. Annals of Oncology. 2005; 16(4):655-63. [DOI:10.1093/annonc/mdi110] [PMID]

[25] Ernst E. Prevalence of use of complementary/alternative medicine: a systematic review. Bulletin of the World Health Organization. 2000; 78(2):258-66.

[26] Tehrani BS, Asgharifard H, Haghdoust A, Barghamadi M, Mohammad HN. The use of Complementary/Alternative Medicine among the general population in Tehran, Iran. 2008; 7(4): 355-62.

[27] Sadighi J, Maftoon F, Ziai S. Herbal medicine: Knowledge, attitude and practice in Tehran. Journal of Medicinal Plants. 2005 1(13):60-7.

[28] World Health Organization. National policy on traditional medicine and regulation of herbal medicines: Report of a WHO global survey. Geneva: World Health Organization; 2005.

[29] Gupta R. Medicinal and Aromatic plants. Patparganj, New Delhi: CBS publishers and distributors. 2010; 234:499.

[30] Kassahun BM, Yosef WB, Mekonnen SA. Performance of lemon Verbena (Aloysia triphylla L.) for morphological, economic and chemical traits in Ethiopia. American-Eurasian Journal of Agricultural and Environmental Sciences. 2013; 13(11):1576-81.

[31] Mojab F, Javidnia K, Zarghi A, Yamohammadi M. Essentia oil of Lippia citriodora HBK (Verbenaceae). Journal of Medicinal Plants. 2002; 4(4):41-6.

[32] Shahhoseini R, Ghorbani H, Karimi SR, Estaji A, Moghaddam M. Qualitative and quantitative changes in the essential oil of lemon verbena (Lippia citriodora) as affected by drying condition. Drying Technology. 2013; 31(9):1020-8. [DOI:10.1080/07373 937.2013.771649] 
[33] Carnat A, Carnat A, Fraisse D, Lamaison J. The aromatic and polyphenolic composition of lemon verbena tea. Fitoterapia. 1999; 70(1):44-9. [DOI:10.1016/S0367-326X(98)00016-1]

[34] Sarris J, Byrne GJ. A systematic review of insomnia and complementary medicine. Sleep Medicine Reviews. 2011; 15(2):99106. [DOI:10.1016/j.smrv.2010.04.001] [PMID]

[35] Nazem-Ekbatani N, Tavoni S, Haghani H, Gharayagh-Zandi S. Evaluation of Satisfaction fromValeirain for Treatment of Menopause Sleep Disorder. Journal of Arak University of Medical Sciences. 2012; 15 (5):49-57.

[36] Buysse DJ, Reynolds CF, Monk TH, Berman SR, Kupfer DJ. The Pittsburgh Sleep Quality Index: A new instrument for psychiatric practice and research. Psychiatry Research. 1989; 28(2):193-213. [DOI:10.1016/0165-1781(89)90047-4]

[37] Tsay S-L, Cho Y-C, Chen M-l. Acupressure and transcutaneous electrical acupoint stimulation in improving fatigue, sleep quality and depression in hemodialysis patients. The American Journal of Chinese Medicine. 2004; 32(03):407-16. [DOI:10.1142/ S0192415X04002065] [PMID]

[38] Backhaus J, Junghanns K, Broocks A, Riemann D, Hohagen F. Test-retest reliability and validity of the Pittsburgh Sleep Quality Index in primary insomnia. Journal of Psychosomatic Research. 2002; 53(3):737-40. [DOI:10.1016/S0022-3999(02)00330-6]

[39] Carpenter JS, Andrykowski MA. Psychometric evaluation of the Pittsburgh sleep quality index. Journal of Psychosomatic Research. 1998; 45(1):5-13. [DOI:10.1016/S0022-3999(97)00298-5]

[40] Dadashpoor A, Mohammadi R. Investigating effect of a period of water exercise on sleep quality in male elders. Journal of Shahid Sadoughi University of Medical Sciences. 2013; 21(3):300-10.

[41] Hossein-Abadi R, Nowrouzi K, Pouresmaili R, Karimloo M, Maddah SSB. Acupoint massage in improving sleep quality of older adults. Archives of Rehabilitation. 2008; 9(2):8-14.

[42] Moghadam K, Farmahini Farahani M, Sajadi M, Sarmadian $\mathrm{H}$, Norouzi Zamenjani M, Heydari HR. The effect of lavender oil aromatherapy on sleep quality of the intensive care unit nurses. Complementary Medicine Journal of Arak. 2016; 5(4):1303-13.

[43] Nasiri K, Jafari M, Eyvanbaga R, Savadpoor M, Seyyedrasooli A. CompComparing the effects of reflexology and foot bath on sleep quality. Complementary Medicine Journal of faculty of Nursing \& Midwifery. 2015; 5(1):1077-90.

[44] Ekbatani N, Taavoni S, Haghani H. The effect of valerian on sleep component among menopausal women. Journal of Kerman University of Medical Sciences. 2012; 19(2):190. 